\title{
SYMMETRY RESULTS FOR STABLE AND MONOTONE SOLUTIONS TO FIBERED SYSTEMS OF PDES
}

\author{
SERENA DIPIERRO, ANDREA PINAMONTI
}

\begin{abstract}
We study the symmetry properties for solutions of elliptic systems of the type

$$
\left\{\begin{array}{l}
-\operatorname{div}\left(a_{1}\left(x,\left|\nabla u^{1}\right|(X)\right) \nabla u^{1}(X)\right)=F_{1}\left(x, u^{1}(X), \ldots, u^{n}(X)\right), \\
\vdots \\
-\operatorname{div}\left(a_{n}\left(x,\left|\nabla u^{n}\right|(X)\right) \nabla u^{n}(X)\right)=F_{n}\left(x, u^{1}(X), \ldots, u^{n}(X)\right),
\end{array}\right.
$$

where $x \in \mathbb{R}^{m}$ with $1 \leq m<N, X=(x, y) \in \mathbb{R}^{m} \times \mathbb{R}^{N-m}$, and $F_{1}, \ldots, F_{n}$ are the derivatives with respect to $\xi^{1}, \ldots, \xi^{n}$ of some $F=F\left(x, \xi^{1}, \ldots, \xi^{n}\right)$ such that for any $i=1, \ldots, n$ and any fixed $\left(x, \xi^{1}, \ldots, \xi^{i-1}, \xi^{i+1}, \ldots, \xi^{n}\right) \in \mathbb{R}^{m} \times \mathbb{R}^{n-1}$ the map $\xi^{i} \rightarrow F\left(x, \xi^{1}, \ldots, \xi^{i}, \ldots, \xi^{n}\right)$ belongs to $C^{2}(\mathbb{R})$. We obtain a Poincaré-type formula for the solutions of the system and we use it to prove a symmetry result both for stable and for monotone solutions.
\end{abstract}

\section{INTRODUCTION}

In this paper we deal with symmetry results for solutions to the following system of partial differential equations defined in an open subset $\Omega$ of $\mathbb{R}^{N}$

$$
\left\{\begin{array}{l}
-\operatorname{div}\left(a_{1}\left(x,\left|\nabla u^{1}\right|(X)\right) \nabla u^{1}(X)\right)=F_{1}\left(x, u^{1}(X), \ldots, u^{n}(X)\right), \\
\vdots \\
-\operatorname{div}\left(a_{n}\left(x,\left|\nabla u^{n}\right|(X)\right) \nabla u^{n}(X)\right)=F_{n}\left(x, u^{1}(X), \ldots, u^{n}(X)\right) .
\end{array}\right.
$$

Here $x \in \mathbb{R}^{m}$ with $1 \leq m<N, X=(x, y) \in \mathbb{R}^{m} \times \mathbb{R}^{N-m}$, and $F_{1}, \ldots, F_{n}$ are the derivatives with respect to $\xi^{1}, \ldots, \xi^{n}$ of some $F=F\left(x, \xi^{1}, \ldots, \xi^{n}\right)$ such that, for any $i=1, \ldots, n$ and any fixed $\left(x, \xi^{1}, \ldots, \xi^{i-1}, \xi^{i+1}, \ldots, \xi^{n}\right) \in \mathbb{R}^{m} \times \mathbb{R}^{n-1}$, the map $\xi^{i} \rightarrow$ $F\left(x, \xi^{1}, \ldots, \xi^{i}, \ldots, \xi^{n}\right)$ belongs to $C^{2}(\mathbb{R})$. We also assume that

- $a_{i} \in L^{\infty}\left(\mathbb{R}^{m} \times\left[\alpha_{-}, \alpha_{+}\right]\right)$, for any $\alpha_{+}>\alpha_{-}>0, i=1, \ldots, n$;

- for any fixed $t \in(0,+\infty)$ and any $i=1, \ldots, n, \inf _{x \in \mathbb{R}^{m}} a_{i}(x, t)>0$;

- for any fixed $x \in \mathbb{R}^{m}$ and any $i=1, \ldots, n$, the map $t \rightarrow a_{i}(x, t)$ is $C^{1}$ on $(0,+\infty)$.

The physical motivation for (1) comes from "fibered", or "stratified" media: namely, the medium, say $\Omega \subset \mathbb{R}^{N}$, is nonhomogeneous, but this nonhomogeneity only occurs in

Key words and phrases. Elliptic systems, fibered media, monotone solutions, stable solutions, phase separation, Poincaré-type inequality.

The first author has been supported by FIRB "Project Analysis and Beyond" and by CAPDE-Anillo ACT-125. The second author has been supported by MIUR, Italy, GNAMPA of INDAM and by Fondazione CaRiPaRo Project "Nonlinear Partial Differential Equations: models, analysis, and control-theoretic problems". 
lower dimensional slices (here, the medium is supposed to be homogeneous with respect to $y \in \mathbb{R}^{N-m}$ and nonhomogeneous with respect to $\left.x \in \mathbb{R}^{m}\right)$.

Systems similar to (11) have been studied in [5]. Precisely, the authors considered the following system

$$
\left\{\begin{array}{l}
\Delta u=u v^{2} \\
\Delta v=v u^{2} \\
u, v>0
\end{array}\right.
$$

which arises in phase separation for multiple states Bose-Einstein condensates. They proved that there exists a solution to (2) in $\mathbb{R}$, which is nondegenerate and reflectionally symmetric, namely that there exists $x_{0} \in \mathbb{R}$ such that $u\left(x-x_{0}\right)=v\left(x-x_{0}\right)$. Moreover, they obtained a result that may be seen as the analogue of a famous conjecture of De Giorgi for problem (2) in dimension 2, that is they proved that monotone solutions of (2) in $\mathbb{R}^{2}$ (see Definition 1.2 below) have one-dimensional symmetry under the additional growth condition

$$
u(x)+v(x) \leq C(1+|x|) .
$$

On the other hand, in [29], it has been proved that the linear growth is the lowest possible for solutions to (2); in other words, if there exists $\alpha \in(0,1)$ such that

$$
u(x)+v(x) \leq C(1+|x|)^{\alpha},
$$

then $u=v \equiv 0$.

In [6] the authors replaced the monotonicity condition by the stability of the solutions (which is a weaker assumption), showing that the above mentioned one-dimensional symmetry still holds in $\mathbb{R}^{2}$. Moreover, they proved that there exist solutions to (2) which do not satisfy the growth condition (3), constructing solutions with polynomial growth.

We mention the paper [37, where the author showed that, for any $n \geq 2$, a solution to (2) which is a local minimizer and satisfies the growth condition (3) has one-dimensional symmetry.

In [23] it is proved that the symmetry result stated in [5] holds also for a more general class of nonlinearities.

Finally, in [16, the author considered a class of quasilinear (possibly degenerate) elliptic systems in $\mathbb{R}^{n}$ and proved that, under suitable assumptions, the solutions have one-dimensional symmetry, showing that the results obtained in [5, 6, 23] hold in a more general setting. We also refer the reader to [18, where symmetry results for systems driven by non local operators are studied.

Results similar to the ones described above are also well-understood in the case of one equation. In particular, in low dimensions, De Giorgi conjecture on the flatness of level sets of standard phase transition (15]) has been proved, see [2, 3, 4, 24, 25. Later, Savin in [31] showed that the conjecture is true up to dimension 8 under an additional hypothesis on the behaviour of the solution at infinity. Finally, in dimension $n \geq 9$ Del Pino, Kowalczyk and Wei constructed a solution to the Allen-Cahn equation which is monotone in one direction but not one-dimensional, see [14].

It is also worth noticing that an analogous of the De Giorgi conjecture has been studied for more general operators. In particular, we mention 20, where quasilinear operators of p-Laplacian and curvature type are considered, and [ $[$, 33], where the authors proved a 
similar De Giorgi-type result for an equation involving the fractional Laplacian in dimension $n=2$; see also [7, 9, 10] for further extensions.

First of all, we give the following definition:

Definition 1.1. An $n$-uple $\left(u^{1}, \ldots, u^{n}\right)$ is said to be a weak solution of (1) if, for any $\psi=\left(\psi^{1}, \ldots, \psi^{n}\right) \in C_{c}^{\infty}\left(\Omega, \mathbb{R}^{n}\right)$,

$$
\int_{\Omega}\left\langle a_{i}\left(x,\left|\nabla u^{i}\right|(X)\right) \nabla u^{i}(X), \nabla \psi^{i}(X)\right\rangle \mathrm{d} X=\int_{\Omega} F_{i}\left(x, u^{1}, \ldots, u^{n}\right) \psi^{i}(X) \mathrm{d} X, \quad i=1, \ldots, n .
$$

In order to state our results we start pointing out our assumptions. In particular, from now on we will always assume that every weak solution $\left(u^{1}, \ldots, u^{n}\right)$ of (1) is such that 1

$$
\begin{aligned}
& u^{i} \in C^{1}(\Omega) \cap C^{2}\left(\Omega \cap\left\{\nabla u^{i} \neq 0\right\}\right) \cap L^{\infty}(\Omega) \\
& \text { and } \nabla u^{i} \in L^{\infty}\left(\Omega, \mathbb{R}^{N}\right) \cap W_{l o c}^{1,2}\left(\Omega, \mathbb{R}^{N}\right), \quad i=1, \ldots, n .
\end{aligned}
$$

Moreover, we will also assume that, for every $k=1, \ldots, n$, the map

$$
\mathcal{A}^{k}: \mathbb{R}^{m} \times\left(\mathbb{R}^{N} \backslash\{0\}\right) \longrightarrow \operatorname{Mat}(N \times N)
$$

defined by 2

$$
\mathcal{A}_{i j}^{k}=\mathcal{A}_{i j}^{k}(x, \eta):=a_{k}(x,|\eta|) \delta_{i j}+\frac{\partial a_{k}}{\partial t}(x,|\eta|) \frac{\eta_{i} \eta_{j}}{|\eta|}, \quad 1 \leq i, j \leq N
$$

is such that 3

$$
\begin{aligned}
& (x, y)=X \rightarrow \mathcal{A}^{i}\left(x, \nabla u^{i}(X)\right) \text { belongs to } L^{\infty}\left(\left\{\nabla u^{i} \neq 0\right\} \cap B_{R}\right) \\
& \text { for any } R>0 \text { and any } i=1, \ldots, n .
\end{aligned}
$$

The following definition was introduced in [23].

Definition 1.2. A solution $\left(u^{1}, \ldots, u^{n}\right)$ of (11) is said to be $F$-monotone if

i) for every $i \in\{1, \ldots, n\}, \partial_{y_{N-m}} u^{i} \neq 0$ in $\Omega$,

ii) for $i<j$, we have $F_{i j} \partial_{y_{N-m}} u^{i} \partial_{y_{N-m}} u^{j} \geq 0$.

As it is customary in this setting, we recall the notion of stability:

Definition 1.3. A solution $\left(u^{1}, \ldots, u^{n}\right)$ of (1) is said to be stable if

$$
\begin{aligned}
\sum_{i=1}^{n} \int_{\Omega}\left\langle\mathcal{A}^{i}\left(x, \nabla u^{i}(X)\right) \nabla \psi^{i}(X), \nabla \psi^{i}(X)\right\rangle \mathrm{d} X \\
\quad-\sum_{i, j=1}^{n} \int_{\Omega} F_{i j}\left(x, u^{1}, \ldots, u^{n}\right) \psi^{i}(X) \psi^{j}(X) \mathrm{d} X \geq 0,
\end{aligned}
$$

for any $\psi=\left(\psi^{1}, \ldots, \psi^{n}\right) \in C_{c}^{\infty}\left(\Omega, \mathbb{R}^{n}\right)$.

\footnotetext{
${ }^{1}$ At the end of this paper we present some explicit cases in which these assumptions are fulfilled.

${ }^{2}$ Here, as usual, $\operatorname{Mat}(N \times N)$ denotes the vector space of real $N \times N$ matrices.

${ }^{3}$ Let us observe that condition (6) is implied if, for example, $\frac{\partial a_{k}}{\partial t} \in L^{\infty}\left(\mathbb{R}^{m} \times[\alpha-, \alpha+]\right)$, for all $\alpha_{+}>$ $\alpha_{-}>0$.
} 
Let us note that (11) represents the Euler-Lagrange system associated to a suitable energy functional $I$ (see the appendix). In particular, the notion of stability given in Definition 1.3 states that $I$ has positive (formal) second variation (we refer to [2, 3, 20] for more details).

According to [11, 20, 34], for every fixed $x \in \mathbb{R}^{m}$ and $c \in \mathbb{R}$, we define

$$
H_{u}=H_{u, x, c}:=\left\{y \in \mathbb{R}^{N-m} \mid u(x, y)=c\right\}
$$

and

$$
L_{u}=L_{u, x, c}:=\left\{y \in H_{u} \mid \nabla_{y} u(x, y) \neq 0\right\} .
$$

We also define

$$
\begin{array}{ll}
\mathcal{R}_{u}:=\left\{(x, y) \in \Omega \mid \nabla_{y} u(x, y) \neq 0\right\}, & \mathcal{S}_{u}:=\sum_{i=1}^{m} \sum_{j=1}^{N-m}\left(u_{x_{i} y_{j}}\right)^{2}-\left.\left|\nabla_{x}\right| \nabla_{y} u\right|^{2}, \\
\mathcal{T}_{u}:=\sum_{j=1}^{N-m}\left\langle\nabla u, \nabla u_{y_{j}}\right\rangle^{2}-\left\langle\nabla u, \nabla\left|\nabla_{y} u\right|\right\rangle^{2}, & \mathcal{U}_{u}:=\sum_{j=1}^{N-m}\left|\nabla u_{y_{j}}\right|^{2}-\left.|\nabla| \nabla_{y} u\right|^{2} .
\end{array}
$$

We recall that the tangential gradient along $L_{u}, \nabla_{L}$, is defined for every $\bar{y} \in L_{u}$ and any $G: \mathbb{R}^{N-m} \longrightarrow \mathbb{R}$ smooth in the vicinity of $\bar{y}$ as

$$
\nabla_{L} G(\bar{y}):=\nabla_{y} G(\bar{y})-\left\langle\nabla_{y} G(\bar{y}), \frac{\nabla_{y} u(x, \bar{y})}{\left|\nabla_{y} u(x, \bar{y})\right|}\right\rangle \frac{\nabla_{y} u(x, \bar{y})}{\left|\nabla_{y} u(x, \bar{y})\right|},
$$

and since $L_{u}$ is a smooth $(N-m-1)$-manifold we define, for every $y \in L_{u}$, the length of the second fundamental form by

$$
\mathcal{K}_{u}(x, y):=\sqrt{\sum_{j=1}^{N-m-1} k_{j}^{2}(x, y)},
$$

where $k_{1, u}(x, y), \ldots, k_{N-m-1, u}(x, y)$ are the principal curvatures of $L_{u}$.

We are now in position to state our main results. We establish first a geometric inequality, which involves the tangential gradients and the curvatures of the level sets of the solution. This inequality holds in every open set $\Omega \subset \mathbb{R}^{N}$.

Theorem 1.4. Let $\left(u^{1}, \ldots, u^{n}\right)$ be a weak stable solution of (1) satisfying (5).

Then, for each $\psi=\left(\psi^{1}, \ldots, \psi^{n}\right) \in C_{c}^{\infty}\left(\Omega, \mathbb{R}^{n}\right)$, we have

$$
\begin{aligned}
& \sum_{k=1}^{n} \int_{\mathcal{R}_{u^{k}}}\left(\sum_{j=1}^{N-m}\left\langle\mathcal{A}^{k} \nabla u_{y_{j}}^{k}, \nabla u_{y_{j}}^{k}\right\rangle-\left\langle\mathcal{A}^{k} \nabla\left|\nabla_{y} u^{k}\right|, \nabla\left|\nabla_{y} u^{k}\right|\right\rangle\right)\left(\psi^{k}\right)^{2} \\
& \quad-\sum_{k, j=1, j \neq k}^{n} \int_{\Omega} F_{k j}\left(\left(\psi^{k}\right)^{2}\left\langle\nabla_{y} u^{j}, \nabla_{y} u^{k}\right\rangle-\psi^{j} \psi^{k}\left|\nabla_{y} u^{k}\right|\left|\nabla_{y} u^{j}\right|\right) \\
& \leq \int_{\Omega} \sum_{k=1}^{n}\left\langle\mathcal{A}^{k} \nabla \psi^{k}, \nabla \psi^{k}\right\rangle\left|\nabla_{y} u^{k}\right|^{2} .
\end{aligned}
$$


Moreover,

$$
\begin{aligned}
& \quad \sum_{k=1}^{n} \int_{R_{u^{k}}}\left[a_{k}\left(x,\left|\nabla u^{k}\right|\right)\left(\mathcal{S}_{u^{k}}+\mathcal{K}_{u^{k}}^{2}\left|\nabla_{y} u^{k}\right|^{2}+\left.\left|\nabla_{L}\right| \nabla_{y} u^{k}\right|^{2}\right)+\frac{\frac{\partial a_{k}}{\partial t}\left(x,\left|\nabla u^{k}\right|\right)}{\left|\nabla u^{k}\right|} \mathcal{T}_{u^{k}}\right]\left(\psi^{k}\right)^{2} \\
& \leq \int_{\Omega} \sum_{k=1}^{n}\left\langle\mathcal{A}^{k} \nabla \psi^{k}, \nabla \psi^{k}\right\rangle\left|\nabla_{y} u^{k}\right|^{2} \\
& \quad-\sum_{k, j=1, j \neq k}^{n} \int_{\Omega} F_{k j}\left(\left(\psi^{k}\right)^{2}\left|\nabla_{y} u^{k}\right|\left|\nabla_{y} u^{j}\right|-\psi^{j} \psi^{k}\left\langle\nabla_{y} u^{i}, \nabla_{y} u^{k}\right\rangle\right) .
\end{aligned}
$$

Next, we state our symmetry results both for stable and for monotone solutions to (1). In the proof of the subsequent theorems, we will use the geometric inequality in (8) with $\Omega=\mathbb{R}^{N}$.

Theorem 1.5. Let $\left(u^{1}, \ldots, u^{n}\right)$ be a weak stable solution of (1) in the whole $\mathbb{R}^{N}$ satisfying (5). Let us also assume that there exist non-zero functions $\theta^{i} \in C^{1}\left(\mathbb{R}^{N}\right), i=1, \ldots, n$, which do not change sign, such that for all $i, j$ with $1 \leq i<j \leq n$ it holds

$$
F_{i j}\left(x, u^{1}(X), \ldots, u^{n}(X)\right) \theta^{i}(X) \theta^{j}(X) \geq 0, \quad \forall X \in \mathbb{R}^{N} .
$$

Moreover, we assume that, for any $i=1, \ldots, n, \mathcal{A}^{i}\left(x, \nabla u^{i}(X)\right)$ satisfies (6)), it is positive definite at almost any $X \in \mathbb{R}^{N}$ and there exist $C_{1}, \ldots, C_{n} \geq 1$ such that the largest eigenvalue $\overline{\mathcal{A}}^{i}(X)$ of $\mathcal{A}^{i}\left(x, \nabla u^{i}(X)\right)$ satisfies

$$
\int_{B_{R}} \overline{\mathcal{A}}^{i}(X)\left|\nabla u^{i}(X)\right|^{2} \mathrm{~d} X \leq C_{i} R^{2}
$$

for any $R \geq \max \left\{C_{1}, \ldots, C_{n}\right\}$.

Then, for each $i=1, \ldots, n$, there exist $\bar{u}^{i}: \mathbb{R}^{m} \times \mathbb{R} \longrightarrow \mathbb{R}$ and $\omega_{i}: \mathbb{R}^{m} \longrightarrow \mathbb{S}^{N-m-1}$ such that

$$
u^{i}(X)=u^{i}(x, y)=\bar{u}^{i}\left(x,\left\langle\omega_{i}(x), y\right\rangle\right)
$$

for any $(x, y) \in \mathbb{R}^{m} \times \mathbb{R}^{N-m}$. Moreover, each $\omega_{i}(x)$ is constant in any connected component of $\left\{\nabla_{y} u^{i} \neq 0\right\}$.

Theorem 1.6. Let $\left(u^{1}, \ldots, u^{n}\right)$ be a weak $F$-monotone solution of (1) in the whole $\mathbb{R}^{N}$ satisfying (5). We assume that, for any $i=1, \ldots, n, \mathcal{A}^{i}\left(x, \nabla u^{i}(X)\right)$ satisfies (6) , it is positive definite at almost any $X \in \mathbb{R}^{N}$ and $\overline{\mathcal{A}}^{i}(X)$ satisfies (10).

Then, there exist $\bar{u}^{i}: \mathbb{R}^{m} \times \mathbb{R} \longrightarrow \mathbb{R}$ and $\omega_{i} \in \mathbb{S}^{N-m-1}$ such that

$$
u^{i}(X)=u^{i}(x, y)=\bar{u}^{i}\left(x,\left\langle\omega_{i}, y\right\rangle\right)
$$

for any $(x, y) \in \mathbb{R}^{m} \times \mathbb{R}^{N-m}$.

If, in addition, there exists $U \subseteq \mathbb{R}^{m} \times \mathbb{R}^{n}$ open such that, for every $j, k=1, \ldots, n$, $F_{j k}>0$ (or $F_{j k}<0$ ) in $U$, then $\omega_{j}=\omega_{k}=\omega$.

Some comments are now in order. We start recalling that a result similar to the one in Theorem 1.4 has been proved in [11, 12, 19, 20, 32, 34, 35] in the case of one equation 
and in [23, 17] for particular systems of equations. Precisely, a geometric inequality like the one in (8) has been obtained in [23] in the case

$$
a_{i}(x, t)=1, \quad F_{i}\left(x, u^{1}, \ldots, u^{n}\right)=F_{i}\left(u^{1}, \ldots, u^{n}\right),
$$

and in [17], for $n=2$, in the case

$$
a_{i}(x, t)=a_{i}(t), \quad F_{i}\left(x, u^{1}, u^{2}\right)=F_{i}\left(u^{1}, u^{2}\right) .
$$

We also mention the papers [21, 22, 30], where an inequality similar to the one obtained in Theorem 1.4 has been established for solutions to semilinear problems in Riemannian and sub-Riemannian manifolds.

We remark that Theorems 1.5 and 1.6 generalize to fibered media the results contained in 23, 17] and allow us to take into account more general systems than the ones considered in [23, 17] (see also the appendix for some explicit examples).

The paper is organized as follows. In the next section we prove the geometric inequality in Theorem 1.4. Sections 3 and 4 are devoted to the proof of Theorems 1.5 and 1.6. Finally, there is an Appendix, which contains some comments on the assumptions made in our theorems.

\section{A GeOmetric inequality: Proof of Theorem 1.4}

Aim of this section is to prove Theorem 1.4. We recall first the following lemma, which has been proved in [11, 34, 35].

Lemma 2.1. For any $u \in C^{2}(\Omega)$, the following equalities hold

$$
\begin{aligned}
& \sum_{j=1}^{N-m}\left\langle\mathcal{A} \nabla u_{y_{j}}, \nabla u_{y_{j}}\right\rangle-\left\langle\mathcal{A} \nabla\left|\nabla_{y} u\right|, \nabla\left|\nabla_{y} u\right|\right\rangle=a(x,|\nabla u|) \mathcal{U}_{u}+\frac{\frac{\partial a}{\partial t}(x,|\nabla u|)}{|\nabla u|} \mathcal{T}_{u} \quad \text { on } \mathcal{R}_{u}, \\
& \mathcal{U}_{u}-\mathcal{S}_{u}=\sum_{i, j=1}^{N-m}\left(u_{y_{i} y_{j}}\right)^{2}-\left.\left|\nabla_{y}\right| \nabla_{y} u\right|^{2}=\mathcal{K}_{u}^{2}\left|\nabla_{y} u\right|^{2}+\left|\nabla_{L}\right| \nabla_{y} u \|^{2} \quad \text { on } \mathcal{R}_{u} . \\
& \text { Moreover, } \mathcal{S}_{u}, \mathcal{T}_{u} \geq 0 \text { on } \mathcal{R}_{u} .
\end{aligned}
$$

In the sequel, we will need also the following result.

Proposition 2.2. Let $\left(u^{1}, \ldots, u^{n}\right)$ be a weak solution of (11) satisfying (5). Suppose that, for each $i=1, \ldots, n, \mathcal{A}^{i}$ verifies (6).

Then, for every $j=1, \ldots, N-m$, the family $\left(u_{y_{j}}^{1}, \ldots, u_{y_{j}}^{n}\right)$ is a weak solution of the following system

$$
\left\{\begin{array}{l}
-\operatorname{div}\left(\mathcal{A}^{1}\left(x, \nabla u^{1}(X)\right) \nabla u_{y_{j}}^{1}(X)\right)=\sum_{i=1}^{n} F_{1 i}\left(x, u^{1}(X), \ldots, u^{n}(X)\right) u_{y_{j}}^{i}(X), \\
\vdots \\
-\operatorname{div}\left(\mathcal{A}^{n}\left(x, \nabla u^{n}(X)\right) \nabla u_{y_{j}}^{n}(X)\right)=\sum_{i=1}^{n} F_{n i}\left(x, u^{1}(X), \ldots, u^{n}(X)\right) u_{y_{j}}^{i}(X) .
\end{array}\right.
$$


Proof. We need to prove that, for every $\psi=\left(\psi^{1}, \ldots, \psi^{n}\right) \in C_{c}^{\infty}\left(\Omega, \mathbb{R}^{n}\right)$, the following equalities hold (we drop for short the dependence of $\mathcal{A}^{i}$ ):

$$
\left\{\begin{array}{l}
\int_{\Omega}\left\langle\mathcal{A}^{1} \nabla u_{y_{j}}^{1}(X), \nabla \psi^{1}(X)\right\rangle=\sum_{i=1}^{n} \int_{\Omega} F_{1 i}\left(x, u^{1}(X), \ldots, u^{n}(X)\right) u_{y_{j}}^{i}(X) \psi^{1}(X), \\
\vdots \\
\int_{\Omega}\left\langle\mathcal{A}^{n} \nabla u_{y_{j}}^{n}(X) \nabla \psi^{n}(X)\right\rangle=\sum_{i=1}^{n} \int_{\Omega} F_{n i}\left(x, u^{1}(X), \ldots, u^{n}(X)\right) u_{y_{j}}^{i}(X) \psi^{n}(X) .
\end{array}\right.
$$

To this end, we fix $i \in\{1, \ldots, N-m\}$, and we use (44) with $\left(\psi_{y_{i}}^{1}, \ldots, \psi_{y_{i}}^{n}\right)$ as test functions. Hence (dropping for short the dependence of $F_{j}$ and $a_{j}$ ), we have

$$
\int_{\Omega} F_{j} \psi_{y_{i}}^{j}=\int_{\Omega}\left\langle a_{j} \nabla u^{j}, \nabla \psi_{y_{i}}^{j}\right\rangle=-\int_{\Omega}\left\langle\left(a_{j} \nabla u^{j}\right)_{y_{i}}, \nabla \psi^{j}\right\rangle=-\int_{\Omega}\left\langle\mathcal{A}^{j} \nabla u_{y_{i}}, \nabla \psi^{j}\right\rangle .
$$

Moreover,

$$
\int_{\Omega} F_{j} \psi_{y_{i}}^{j}=-\int_{\Omega}\left(F_{j}\right)_{y_{i}} \psi^{j}=-\sum_{k=1}^{n} \int_{\Omega} F_{j k} u_{y_{i}}^{k} \psi^{j},
$$

and putting together (12) and (13) we get the thesis.

Remark 2.3. By an easy density argument (see [12] for the details), we have that (11) holds for any $\psi=\left(\psi^{1}, \ldots, \psi^{n}\right) \in W_{0}^{1,2}\left(\Omega, \mathbb{R}^{n}\right)$.

Proof of Theorem 1.4: Let us fix $1 \leq j \leq N-m$ and $\psi=\left(\psi^{1}, \ldots, \psi^{n}\right) \in C_{c}^{\infty}\left(\Omega, \mathbb{R}^{n}\right)$. Then, by (5), we have that $\varphi^{i}:=u_{y_{j}}^{i}\left(\psi^{i}\right)^{2} \in W_{0}^{1,2}(\Omega)$. Hence, by Remark 2.3, we can use $\varphi=\left(\varphi^{1}, \ldots, \varphi^{n}\right)$ as a test function in (11). It follows that (dropping for short the dependence of $\mathcal{A}^{i}$ and $F_{i j}$ ), for any $k=1, \ldots n$,

$$
\int_{\Omega}\left\langle\mathcal{A}^{k} \nabla u_{y_{j}}^{k}, \nabla\left(u_{y_{j}}^{k}\left(\psi^{k}\right)^{2}\right)\right\rangle=\sum_{i=1}^{n} \int_{\Omega} F_{k i} u_{y_{j}}^{i} u_{y_{j}}^{k}\left(\psi^{k}\right)^{2} .
$$

Summing over $j$, we have

$$
\sum_{j=1}^{N-m} \int_{\Omega}\left\langle\mathcal{A}^{k} \nabla u_{y_{j}}^{k}, \nabla\left(u_{y_{j}}^{k}\left(\psi^{k}\right)^{2}\right)\right\rangle=\sum_{i=1}^{n} \int_{\Omega} F_{k i}\left(\psi^{k}\right)^{2}\left\langle\nabla_{y} u^{i}, \nabla_{y} u^{k}\right\rangle,
$$

which implies

$$
\begin{aligned}
\int_{\Omega} F_{k k}\left(\psi^{k}\right)^{2}\left|\nabla_{y} u^{k}\right|^{2}= & \sum_{j=1}^{N-m} \int_{\Omega}\left\langle\mathcal{A}^{k} \nabla u_{y_{j}}^{k}, \nabla\left(u_{y_{j}}^{k}\left(\psi^{k}\right)^{2}\right)\right\rangle \\
& \quad-\sum_{i=1, i \neq k}^{n} \int_{\Omega} F_{k i}\left(\psi^{k}\right)^{2}\left\langle\nabla_{y} u^{i}, \nabla_{y} u^{k}\right\rangle .
\end{aligned}
$$

Using Stampacchia's Theorem (see, for istance, [28, Theorem 6.19]), we get

$$
\nabla\left|\nabla_{y} u^{k}\right|=0=\nabla u_{y_{j}}^{k} \quad \text { for a.e. } x \in \mathbb{R}^{m} \text {, and a.e. } y \in \mathbb{R}^{N-m} \text { s.t. } \nabla_{y} u(x, y)=0 .
$$


Hence, summing over $k=1, \ldots, n$ in (14), we obtain

$$
\begin{aligned}
\sum_{k=1}^{n} \int_{\Omega} F_{k k}\left(\psi^{k}\right)^{2}\left|\nabla_{y} u^{k}\right|^{2}=\sum_{k=1}^{n} & \int_{\mathcal{R}_{u^{k}}} \sum_{j=1}^{N-m}\left\langle\mathcal{A}^{k} \nabla u_{y_{j}}^{k}, \nabla\left(u_{y_{j}}^{k}\left(\psi^{k}\right)^{2}\right)\right\rangle \\
& -\sum_{k, i=1, i \neq k}^{n} \int_{\Omega} F_{k i}\left(\psi^{k}\right)^{2}\left\langle\nabla_{y} u^{i}, \nabla_{y} u^{k}\right\rangle .
\end{aligned}
$$

Using $\varphi^{k}:=\left|\nabla_{y} u^{k}\right| \psi^{k}$, with $\psi=\left(\psi^{1}, \ldots, \psi^{n}\right) \in C_{c}^{\infty}\left(\Omega, \mathbb{R}^{n}\right)$, as a test function in (7) (we point out that by the regularity assumptions on $u^{i}$ it follows that $\varphi^{i} \in W_{0}^{1,2}(\Omega)$, $i=1, \ldots, n)$, and recalling (15), we obtain

$$
\begin{aligned}
0 \leq \sum_{k=1}^{n} & \int_{\mathcal{R}_{u^{k}}}\left\langle\mathcal{A}^{k} \nabla\left|\nabla_{y} u^{k}\right|, \nabla\left|\nabla_{y} u^{k}\right|\right\rangle\left(\psi^{k}\right)^{2}+\left\langle\mathcal{A}^{k} \nabla \psi^{k}, \nabla \psi^{k}\right\rangle\left|\nabla_{y} u^{k}\right|^{2} \\
& +2\left\langle\mathcal{A}^{k} \nabla\left|\nabla_{y} u^{k}\right|, \nabla \psi^{k}\right\rangle\left|\nabla_{y} u^{k}\right| \psi^{k} \\
& -\sum_{k, j=1, j \neq k}^{n} \int_{\Omega} F_{k j}\left|\nabla_{y} u^{k}\right|\left|\nabla_{y} u^{j}\right| \psi^{j} \psi^{k}-\sum_{k=1}^{n} \int_{\Omega} F_{k k}\left|\nabla_{y} u^{k}\right|^{2}\left(\psi^{k}\right)^{2},
\end{aligned}
$$

which together with (16) implies

$$
\begin{aligned}
0 \leq \sum_{k=1}^{n} & \int_{\mathcal{R}_{u^{k}}}\left\langle\mathcal{A}^{k} \nabla\left|\nabla_{y} u^{k}\right|, \nabla\left|\nabla_{y} u^{k}\right|\right\rangle\left(\psi^{k}\right)^{2}+\left\langle\mathcal{A}^{k} \nabla \psi^{k}, \nabla \psi^{k}\right\rangle\left|\nabla_{y} u^{k}\right|^{2} \\
& +\frac{1}{2}\left\langle\mathcal{A}^{k} \nabla\left|\nabla_{y} u^{k}\right|^{2}, \nabla\left(\psi^{k}\right)^{2}\right\rangle \\
& +\sum_{k, j=1, j \neq k}^{n} \int_{\Omega} F_{k j}\left(\left(\psi^{k}\right)^{2}\left\langle\nabla_{y} u^{j}, \nabla_{y} u^{k}\right\rangle-\psi^{j} \psi^{k}\left|\nabla_{y} u^{k}\right|\left|\nabla_{y} u^{j}\right|\right) \\
& -\sum_{k=1}^{n} \int_{\mathcal{R}_{u^{k}}} \sum_{j=1}^{N-m}\left(\psi^{k}\right)^{2}\left\langle\mathcal{A}^{k} \nabla u_{y_{j}}^{k}, \nabla u_{y_{j}}^{k}\right\rangle \\
& -\sum_{k=1}^{n} \frac{1}{2} \int_{\mathcal{R}_{u^{k}}} \mathcal{A}^{k}\left\langle\nabla\left|\nabla_{y} u^{k}\right|^{2}, \nabla\left(\psi^{k}\right)^{2}\right\rangle .
\end{aligned}
$$

Rewriting the inequality in (17) in a more compact form, we obtain

$$
\begin{aligned}
& 0 \leq \sum_{k=1}^{n} \int_{\mathcal{R}_{u^{k}}} {\left[\left(\left\langle\mathcal{A}^{k} \nabla\left|\nabla_{y} u^{k}\right|, \nabla\left|\nabla_{y} u^{k}\right|\right\rangle-\sum_{j=1}^{N-m}\left\langle\mathcal{A}^{k} \nabla u_{y_{j}}^{k}, \nabla u_{y_{j}}^{k}\right\rangle\right)\left(\psi^{k}\right)^{2}\right.} \\
&\left.+\left\langle\mathcal{A}^{k} \nabla \psi^{k}, \nabla \psi^{k}\right\rangle\left|\nabla_{y} u^{k}\right|^{2}\right] \\
&+\sum_{k, j=1, j \neq k}^{n} \int_{\Omega} F_{k j}\left(\left(\psi^{k}\right)^{2}\left\langle\nabla_{y} u^{j}, \nabla_{y} u^{k}\right\rangle-\psi^{j} \psi^{k}\left|\nabla_{y} u^{k}\right|\left|\nabla_{y} u^{j}\right|\right),
\end{aligned}
$$


which is the first part of the statement. For the second part, we observe that, by Lemma 2.1, we get

$$
\begin{aligned}
& \left\langle\mathcal{A}^{k} \nabla\left|\nabla_{y} u^{k}\right|, \nabla\left|\nabla_{y} u^{k}\right|\right\rangle-\sum_{j=1}^{N-m}\left\langle\mathcal{A}^{k} \nabla u_{y_{j}}^{k}, \nabla u_{y_{j}}^{k}\right\rangle \\
= & -a_{k}\left(x,\left|\nabla u^{k}\right|\right)\left(\mathcal{S}_{u^{k}}+\mathcal{K}_{u^{k}}^{2}\left|\nabla_{y} u^{k}\right|^{2}+\left.\left|\nabla_{L}\right| \nabla_{y} u^{k}\right|^{2}\right)-\frac{\frac{\partial a_{k}}{\partial t}\left(x,\left|\nabla u^{k}\right|\right)}{\left|\nabla u^{k}\right|} \mathcal{T}_{u^{k}} \quad \text { on } \mathcal{R}_{u^{k}} .
\end{aligned}
$$

Therefore, plugging (18), into (17) we get the thesis.

\section{Stable solutions and Proof of Theorem 1.5}

Recalling the definition of stable solutions to (11) given in (7), in this section we will prove Theorem 1.5.

First, we recall the following lemma from [12].

Lemma 3.1. Let $R>0$ and $h: B_{R} \subset \mathbb{R}^{N} \longrightarrow \mathbb{R}$ be a nonnegative measurable function. For any $\rho \in(0, R)$, let

$$
\xi(\rho):=2 \int_{B_{\rho}} h(X) \mathrm{d} X
$$

Then,

$$
\int_{B_{R} \backslash B_{\sqrt{R}}} \frac{h(X)}{|X|^{2}} \mathrm{~d} X \leq \int_{\sqrt{R}}^{R} t^{-3} \xi(t) \mathrm{d} t+\frac{\xi(R)}{R^{2}} .
$$

Proof of Theorem 1.5: In order to prove Theorem 1.5, we use the geometric inequality in (8), with $\Omega=\mathbb{R}^{N}$. Since $\mathcal{A}^{k}$ is positive definite, inequality (8) becomes

$$
\begin{aligned}
& \sum_{k=1}^{n} \int_{\mathcal{R}_{u^{k}}}\left(\sum_{j=1}^{N-m}\left\langle\mathcal{A}^{k} \nabla u_{y_{j}}^{k}, \nabla u_{y_{j}}^{k}\right\rangle-\left\langle\mathcal{A}^{k} \nabla\left|\nabla_{y} u^{k}\right|, \nabla\left|\nabla_{y} u^{k}\right|\right\rangle\right)\left(\psi^{k}\right)^{2} \\
& \quad-\sum_{k, j=1, j \neq k}^{n} \int_{\mathbb{R}^{N}} F_{k j}\left(\left(\psi^{k}\right)^{2}\left\langle\nabla_{y} u^{j}, \nabla_{y} u^{k}\right\rangle-\psi^{j} \psi^{k}\left|\nabla_{y} u^{k}\right|\left|\nabla_{y} u^{j}\right|\right) \\
& \leq \sum_{k=1}^{n} \int_{\mathbb{R}^{N}} \overline{\mathcal{A}}^{k}\left|\nabla \psi^{k}\right|^{2}\left|\nabla_{y} u^{k}\right|^{2} .
\end{aligned}
$$

By Lemma 3.1 in [11], we have

$$
\sum_{j=1}^{N-m}\left\langle\mathcal{A}^{k} \nabla u_{y_{j}}^{k}, \nabla u_{y_{j}}^{k}\right\rangle-\left\langle\mathcal{A}^{k} \nabla\left|\nabla_{y} u^{k}\right|, \nabla\left|\nabla_{y} u^{k}\right|\right\rangle \geq 0, \quad k=1, \ldots, n .
$$

Moreover, by (9), we have that there exist non-zero functions $\theta^{1}, \ldots, \theta^{n} \in C^{1}\left(\mathbb{R}^{N}\right)$ with constant sign such that

$$
F_{i j} \theta^{i} \theta^{j} \geq 0, \quad \forall i, j \in\{1, \ldots, n\}, i<j .
$$


For any $R>1$, we define $\eta_{R}: \mathbb{R}^{N} \longrightarrow \mathbb{R}$ as

$$
\eta_{R}(X):=\left\{\begin{array}{lc}
1 & \text { if } X \in B_{\sqrt{R}}, \\
2 \frac{\log R-\log |X|}{\log R} & \text { if } X \in B_{R} \backslash B_{\sqrt{R}}, \\
0 & \text { if } X \in \mathbb{R}^{N} \backslash B_{R},
\end{array}\right.
$$

and consider

$$
\eta_{R}^{i}:=\operatorname{sgn}\left(\theta^{i}\right) \eta_{R}
$$

where $\operatorname{sgn}(x)$ is the Sign function. It follows that, for each $i=1, \ldots, n, \eta_{R}^{i} \in C_{c}^{\infty}\left(B_{R}\right)$, $0<\left|\eta_{R}^{i}(X)\right|<1$ for any $X \in \mathbb{R}^{N}$, and

$$
\left|\nabla \eta_{R}^{i}(X)\right| \leq \frac{\chi_{R}(X)}{2|X| \log R}
$$

where

$$
\chi_{R}(X):= \begin{cases}1 & \text { if } X \in B_{R} \backslash B_{\sqrt{R}} \\ 0 & \text { otherwise. }\end{cases}
$$

Moreover, from (20), we have

$$
F_{i j} \operatorname{sgn}\left(\theta^{i}\right) \operatorname{sgn}\left(\theta^{j}\right) \geq 0, \quad \forall i, j \in\{1, \ldots, n\}, i<j .
$$

Using (21) as a test function in (19), and observing that

$$
F_{k j} \operatorname{sgn}\left(\theta^{k}\right) \operatorname{sgn}\left(\theta^{j}\right)=\operatorname{sgn}\left(F_{k j}\right) F_{k j},
$$

we get

$$
\begin{aligned}
& \quad \sum_{k=1}^{n} \int_{\mathcal{R}_{u^{k}}}\left[\sum_{j=1}^{N-m}\left\langle\mathcal{A}^{k} \nabla u_{y_{j}}^{k}, \nabla u_{y_{j}}^{k}\right\rangle-\left\langle\mathcal{A}^{k} \nabla\left|\nabla_{y} u^{k}\right|, \nabla\left|\nabla_{y} u^{k}\right|\right\rangle\right]\left(\eta_{R}^{k}\right)^{2} \\
& \quad-\sum_{k, j=1, j \neq k}^{n} \int_{\mathbb{R}^{N}}\left(\operatorname{sgn}\left(F_{k j}\right)\left\langle\nabla_{y} u^{j}, \nabla_{y} u^{k}\right\rangle-\left|\nabla_{y} u^{k}\right|\left|\nabla_{y} u^{j}\right|\right) F_{k j} \operatorname{sgn}\left(F_{k j}\right) \eta_{R}^{2} \\
& \leq \frac{1}{4 \log ^{2} R} \sum_{k=1}^{n} \int_{B_{R} \backslash B_{\sqrt{R}}} \frac{\overline{\mathcal{A}}^{k}\left|\nabla_{y} u^{k}\right|^{2}}{|X|^{2}} \\
& \leq C \frac{1}{\log R},
\end{aligned}
$$

where in the last inequality we have used the fact that $\left|\nabla_{y} u\right|^{2} \leq|\nabla u|^{2}$, Lemma 3.1 with $h(X):=\overline{\mathcal{A}}^{k}\left|\nabla_{y} u^{k}\right|^{2}$ and the assumption (10).

Sending $R \rightarrow+\infty$ in (22), we conclude that

$$
\sum_{j=1}^{N-m}\left\langle\mathcal{A}^{k} \nabla u_{y_{j}}^{k}, \nabla u_{y_{j}}^{k}\right\rangle-\left\langle\mathcal{A}^{k} \nabla\left|\nabla_{y} u^{k}\right|, \nabla\left|\nabla_{y} u^{k}\right|\right\rangle=0, \quad \text { a.e. in } \mathcal{R}_{u^{k}}, k=1, \ldots, n,
$$

and

$$
\left(\operatorname{sgn}\left(F_{k j}\right)\left\langle\nabla_{y} u^{j}, \nabla_{y} u^{k}\right\rangle-\left|\nabla_{y} u^{k}\right|\left|\nabla_{y} u^{j}\right|\right) \operatorname{sgn}\left(F_{k j}\right) F_{k j}=0, \quad \text { a.e. in } \mathbb{R}^{N},
$$


for any $k, j=1, \ldots, n$, with $j \neq k$.

By (23) and Corollary 3.2 in [11, we obtain that, for any level set $L$ of $u^{k}$ and any $X \in \mathcal{R}_{u^{k}} \cap L$,

$$
\mathcal{K}_{u^{k}}=0=\left|\nabla_{L}\right| \nabla_{y} u^{k}|| .
$$

Therefore, using Lemma 2.11 in [20], this implies that, for each $k=1, \ldots, n$, there exist $\omega_{k}: \mathbb{R}^{m} \longrightarrow \mathbb{S}^{N-m-1}$ and $\bar{u}^{k}: \mathbb{R}^{m} \times \mathbb{R} \longrightarrow \mathbb{R}$ such that

$$
u^{k}(x, y)=\bar{u}^{k}\left(x,\left\langle\omega_{k}(x), y\right\rangle\right) .
$$

Moreover, by Lemma A.1 in 12 we have that each $\omega_{k}(x)$ is constant in any connected component of $\left\{\nabla_{y} u^{k} \neq 0\right\}$. This concludes the proof.

There are some cases in which the directions $\omega_{1}, \ldots, \omega_{n}$ may be related or may coincide. In fact, as a corollary of Theorem 1.5, we prove that this happens under some additional assumptions on the functions $F_{k j}$. For this, we denote by $\Im\left(u^{1}, \ldots, u^{n}\right)$ the image of the $\operatorname{map}\left(u^{1}, \ldots, u^{n}\right): \mathbb{R}^{N} \rightarrow \mathbb{R}^{n}$, that is

$$
\Im\left(u^{1}, \ldots, u^{n}\right):=\left\{\left(u^{1}(X), \ldots, u^{n}(X)\right), X \in \mathbb{R}^{N}\right\} .
$$

Then, the following symmetry result holds:

Corollary 3.2. Under the assumptions of Theorem 1.5, we assume that, for every $x \in \mathbb{R}^{m}$ and for every $j, k=1, \ldots, n, j \neq k$,

there exist open intervals $I_{1}^{x}, \ldots, I_{n}^{x} \subset \mathbb{R}$ such that $\left(I_{1}^{x} \times \ldots \times I_{n}^{x}\right) \cap \Im\left(u^{1}, \ldots, u^{n}\right) \neq \varnothing$ and $F_{k j}\left(x, \overline{u^{1}}, \ldots, \overline{u^{n}}\right)>0\left(\right.$ or $\left.F_{k j}\left(x, \overline{u^{1}}, \ldots, \overline{u^{n}}\right)<0\right)$

for any $\left(\overline{u^{1}}, \ldots, \overline{u^{n}}\right) \in I_{1}^{x} \times \ldots \times I_{n}^{x}$.

Then there exist $C_{j k}: \mathbb{R}^{N} \longrightarrow \mathbb{R}$ and $D_{j k}: \mathbb{R}^{m} \longrightarrow\{-1,1\}$ such that

$$
\nabla_{y} u^{j}(X)=C_{j k}(X) \nabla_{y} u^{k}(X) \quad \text { and } \quad \omega_{j}(x)=D_{j k}(x) \omega_{k}(x) .
$$

If, in addition, $\mathcal{I}:=\bigcap_{i=1}^{n}\left\{\nabla_{y} u^{i} \neq 0\right\} \neq \varnothing$ is connected, then $\omega_{1} \equiv \ldots \equiv \omega_{n}$ in $\mathcal{I}$.

Proof. By Theorem 1.5, for each $j, k=1, \ldots, n$,

$$
u^{j}(x, y)=\bar{u}^{j}\left(x,\left\langle\omega_{j}(x), y\right\rangle\right), \quad u^{k}(x, y)=\bar{u}^{k}\left(x,\left\langle\omega_{k}(x), y\right\rangle\right),
$$

for some $\bar{u}^{j}, \bar{u}^{k}: \mathbb{R}^{m} \times \mathbb{R} \longrightarrow \mathbb{R}$ and $\omega_{j}, \omega_{k}: \mathbb{R}^{m} \longrightarrow \mathbb{S}^{N-m-1}$.

Now, for any fixed $x \in \mathbb{R}^{m}$, arguing as in the proof of the second part of Theorem 1.8 in [18, one can prove that there exists a non-empty open set $V \subset \mathbb{R}^{N-m}$ such that $u^{i}(x, y) \in I_{i}^{x}$ and $\nabla_{y} u^{i}(x, y) \neq 0$, for all $y \in V$ and $i=1, \ldots, n$.

Therefore, using (24) and (25), we obtain that there exists $y_{*} \in V$ such that

$$
\operatorname{sgn}\left(F_{k j}\right)\left\langle\nabla_{y} u^{j}\left(x, y_{*}\right), \nabla_{y} u^{k}\left(x, y_{*}\right)\right\rangle-\left|\nabla_{y} u^{k}\left(x, y_{*}\right)\right|\left|\nabla_{y} u^{j}\left(x, y_{*}\right)\right|=0,
$$

for any $j, k=1, \ldots, n, j \neq k$. Moreover, from (27), we have that $\nabla_{y} u^{j}\left(x, y_{*}\right)$ is proportional to $\omega_{j}(x)$ and $\nabla_{y} u^{k}\left(x, y_{*}\right)$ is proportional to $\omega_{k}(x)$. Hence, (28) together with (27) implies (26). 
If $\mathcal{I} \neq \varnothing$ is connected, we have that

$$
\omega_{i}(x)=\omega_{i}, \quad i=1, \ldots, n,
$$

because, from Theorem 1.5, we know that each $\omega_{i}$ is constant in any connected component of $\left\{\nabla_{y} u^{i} \neq 0\right\}$.

Now, plugging the functions in (27) into (28), we have that

$$
\left|\partial_{z} \bar{u}^{j}\right|\left|\partial_{z} \bar{u}^{k}\right|\left( \pm\left\langle\omega_{j}, \omega_{k}\right\rangle-1\right)=0, \quad j, k=1, \ldots, n, j \neq k,
$$

where $\partial_{z} \bar{u}^{i}$ denotes the derivative of the function $\bar{u}^{i}$ with respect to the last variable. Therefore, from the last equality, we deduce that, for every $j, k=1, \ldots, n$,

$$
\left\langle\omega_{j}, \omega_{k}\right\rangle= \pm 1, \quad \text { in } \mathcal{I} .
$$

If $\omega_{k}=-\omega_{j}$, we have that $u^{j}(x, y)=\bar{u}^{j}\left(x,\left\langle\omega_{j}, y\right\rangle\right)$ and $u^{k}(x, y)=\bar{u}^{k}\left(x,\left\langle-\omega_{j}, y\right\rangle\right)$; then, we can define $\tilde{u}^{k}(x, y):=\bar{u}^{k}(x,-y)$, and obtain $u^{k}(x, y)=\tilde{u}^{k}\left(x,\left\langle\omega_{j}, y\right\rangle\right)$. This means that we can take $\omega_{j}=\omega_{k}$ up to renaming the function that describes $u^{k}$. Hence, we have that $\omega_{j}=\omega_{k}$ for every $j, k=1, \ldots, n$, and this concludes the proof.

\section{Monotone solutions And proof of Theorem 1.6}

In this section we prove our symmetry result for monotone solutions to (11). First of all, we show that $F$-monotonicity implies stability (see Definitions 1.2 and 1.3).

Proposition 4.1. If $\left(u^{1}, \ldots, u^{n}\right)$ is a $F$-monotone solution of (1), then it is also stable.

Proof. Choosing $\psi^{i}:=\frac{\xi_{i}^{2}}{u_{y_{N-m}}^{i}} \in W_{0}^{1,2}(\Omega)$ in (11), where $\xi_{i} \in C_{c}^{\infty}(\Omega)$ (we explicitly observe that this is possible thanks to Remark 2.3), we obtain

$$
\begin{aligned}
\sum_{j=1}^{n} \int_{\Omega} F_{i j} \frac{u_{y_{N-m}}^{j}}{u_{y_{N-m}}^{i}} \xi_{i}^{2}= & \int_{\Omega}\left\langle\mathcal{A}^{i} \nabla u_{y_{N-m}}^{i}, \frac{\left.\left(\nabla \xi_{i}^{2}\right) u_{y_{N-m}}^{i}-\xi_{i}^{2} \nabla u_{y_{N-m}}^{i}\right\rangle}{\left(u_{y_{N-m}}^{i}\right)^{2}}\right\rangle \\
= & -\int_{\Omega} \frac{\xi_{i}^{2}}{\left(u_{y_{N-m}}^{i}\right)^{2}}\left\langle\mathcal{A}^{i} \nabla u_{y_{N-m}}^{i}, \nabla u_{y_{N-m}}^{i}\right\rangle \\
& +2 \int_{\Omega} \frac{\xi_{i}}{u_{y_{N-m}}^{i}}\left\langle\mathcal{A}^{i} \nabla u_{y_{N-m}}^{i}, \nabla \xi_{i}\right\rangle \\
\leq & \int_{\Omega}\left\langle\mathcal{A}^{i} \nabla \xi_{i}, \nabla \xi_{i}\right\rangle,
\end{aligned}
$$

where in the last inequality we have used the fact that since $\mathcal{A}^{i}$ is positive definite then the following inequality holds for each $a \in \mathbb{R}, v, w \in \mathbb{R}^{N}, i \in\{1, \ldots, n\}$ :

$$
2 a\left\langle\mathcal{A}^{i} v, w\right\rangle-a^{2}\left\langle\mathcal{A}^{i} w, w\right\rangle-\left\langle\mathcal{A}^{i} v, v\right\rangle=\left\langle\mathcal{A}^{i}(v-a w), a w-v\right\rangle \leq 0 .
$$

Summing over $i \in\{1, \ldots, n\}$ in (29), we get

$$
\sum_{i, j=1}^{n} \int_{\Omega} F_{i j} \frac{u_{y_{N-m}}^{j}}{u_{y_{N-m}}^{i}} \xi_{i}^{2} \leq \sum_{i=1}^{n} \int_{\Omega}\left\langle\mathcal{A}^{i} \nabla \xi_{i}, \nabla \xi_{i}\right\rangle, \quad \forall \xi_{i} \in C_{c}^{\infty}(\Omega) .
$$


Moreover,

$$
\begin{aligned}
\sum_{i, j=1}^{n} F_{i j} \frac{u_{y_{N-m}}^{j}}{u_{y_{N-m}}^{i}} \xi_{i}^{2} & =\sum_{i, j=1}^{n} F_{i j} u_{y_{N-m}}^{i} u_{y_{N-m}}^{j} \frac{\xi_{i}^{2}}{\left(u_{y_{N-m}}^{i}\right)^{2}} \\
& =\sum_{i=1}^{n} F_{i i} \xi_{i}^{2}+\sum_{i<j} F_{i j} u_{y_{N-m}}^{i} u_{y_{N-m}}^{j}\left(\frac{\xi_{i}^{2}}{\left(u_{y_{N-m}}^{i}\right)^{2}}+\frac{\xi_{j}^{2}}{\left(u_{y_{N-m}}^{j}\right)^{2}}\right) \\
& \geq \sum_{i, j=1}^{n} F_{i j} \xi_{i} \xi_{j},
\end{aligned}
$$

where in the last inequality we have used the fact that $F_{i j} u_{y_{N-m}}^{i} u_{y_{N-m}}^{j} \geq 0$ if $i<j$. Putting together (30) and (31) we get the thesis.

Proof of Theorem 1.6: By Proposition 4.1, every monotone solution of (1) is also stable. Moreover, the assumption in (9) is verified (it is enough to take $\theta^{i}:=u_{y_{N-m}}^{i}$, $\theta^{j}:=u_{y_{N-m}}^{j}$ which belong to $C^{1}\left(\mathbb{R}^{N}\right)$ thanks to (5D)). Then, the hypotheses of Theorem 1.5 are satisfied, and therefore we conclude that there exist $\bar{u}^{i}: \mathbb{R}^{m} \times \mathbb{R} \longrightarrow \mathbb{R}$ and $\omega_{i} \in \mathbb{S}^{N-m-1}$ such that

$$
u^{i}(X)=u^{i}(x, y)=\bar{u}^{i}\left(x,\left\langle\omega_{i}, y\right\rangle\right)
$$

for any $(x, y) \in \mathbb{R}^{m} \times \mathbb{R}^{N-m}, i \in\{1, \ldots, n\}$.

Let us now assume that there exists $U \subseteq \mathbb{R}^{m} \times \mathbb{R}^{n}$ open such that, for every $j, k=$ $1, \ldots, n, F_{j k}>0\left(\right.$ or $\left.F_{j k}<0\right)$ in $U$. Using (24) and (32), we get

$$
\pm\left|\partial_{z} \bar{u}^{j}\right|\left|\partial_{z} \bar{u}^{k}\right|\left\langle\omega_{j}, \omega_{k}\right\rangle=\left|\partial_{z} \bar{u}^{j}\right|\left|\partial_{z} \bar{u}^{k}\right| \quad \text { in } U
$$

which implies $\left\langle\omega_{j}, \omega_{k}\right\rangle= \pm 1$, and hence $\omega_{j}=\omega_{k}=\omega$ (see the comments at the end of the proof of Corollary 3.2). This concludes the proof.

\section{Appendix A.}

In this appendix we analyze the assumptions made in Section 1 in order to get our symmetry results.

A.1. Optimality of the assumptions. We start observing that the regularity assumptions (5) are fulfilled in a lot of interesting cases. Precisely, let $\left(u^{1}, \ldots, u^{n}\right)$ be a solution of (1) with $u^{i} \in W^{1, p_{i}}(\Omega) \cap L^{\infty}(\Omega)$, and define

$$
b_{i}^{j}\left(x, \nabla u^{i}(X)\right):=a_{i}\left(x,\left|\nabla u^{i}\right|(X)\right) \partial_{j} u^{i}(X), \quad G_{i}(X):=F_{i}\left(x, u^{1}(X), \ldots, u^{n}(X)\right) .
$$


Let us assume that for every $i=1, \ldots, n$

$$
\begin{aligned}
& b_{i}^{j} \in C^{0}\left(\mathbb{R}^{m} \times \mathbb{R}^{N}\right) \cap C^{1}\left(\mathbb{R}^{m} \times \mathbb{R}^{N} \backslash\{0\}\right), \quad j=1, \ldots, N \\
& \sum_{j, k=1}^{N} \frac{\partial b_{i}^{j}}{\partial \eta_{k}}(x, \eta) \xi_{j} \xi_{k} \geq \sigma(k+|\eta|)^{p_{i}-1}|\xi|^{2}, \\
& \sum_{j, k=1}^{N}\left|\frac{\partial b_{i}^{j}}{\partial \eta_{k}}(x, \eta)\right| \leq \Gamma(k+|\eta|)^{p_{i}-2}, \\
& \sum_{j, k=1}^{N}\left|\frac{\partial b_{i}^{j}}{\partial x_{k}}(x, \eta)\right| \leq \Gamma(k+|\eta|)^{p_{i}-2}|\eta|, \\
& \left|G_{i}(X)\right| \leq \Gamma,
\end{aligned}
$$

for all $\eta \in \mathbb{R}^{N} \backslash\{0\}, \xi \in \mathbb{R}^{N}, X \in \mathbb{R}^{N}$, with $p_{i} \geq 2, k \in[0,1], \Gamma, \sigma>0$.

Then, by [16, 27, 36, 26], we conclude that $u^{i} \in C^{1}\left(\mathbb{R}^{N}\right) \cap C^{2}\left(\left\{\nabla u^{i} \neq 0\right\}\right)$ for each $i=1, \ldots, n$.

Moreover, using (2.2.2) in 36] and Theorem 1.1 and Proposition 2.2 in [13, we conclude that also the assumption $u^{i} \in W_{l o c}^{1,2}\left(\mathbb{R}^{N}\right)$ is always verified if either $\left\{\nabla u^{i}=0\right\}=\varnothing$ for $i=1, \ldots, n$ or $1<p<3$.

Therefore, the functions $u^{i}$ satisfy the regularity assumptions in (5) provided the conditions in (33)-(37) hold.

It is interesting to note that, as in the scalar case, the assumption $|\nabla u| \in L^{\infty}(\Omega)$ cannot be removed. Indeed, without such an assumption, one can find a solution which is not one-dimensional, according to the following proposition (see Proposition 3.1 in [20]):

Proposition A.1. Let $k>0$ and $\psi \in C^{1}((k,+\infty))$ satisfying $\dot{\psi}(t)>0$ in $(k,+\infty)$ and $\lim _{t \rightarrow+\infty} \psi(t)=+\infty$. Then, there exists $a \in C^{1}((0,+\infty))$ strictly positive, and $u \in C^{2}\left(\mathbb{R}^{N}\right)$ which is a stable solution of

$$
-\operatorname{div}(a(|\nabla u(X)|) \nabla u(X))=N
$$

and such that $|\nabla u(X)|=\psi(|X|)$ for any $|X|$ suitably large.

Moreover, $u$ does not possess one-dimensional symmetry.

We also mention that, proceeding exactly as in [20, 17], the assumption on the regularity of $F$, i.e. for any $\left(x, \xi^{1}, \ldots, \xi^{i-1}, \xi^{i+1}, \ldots, \xi^{n}\right) \in \mathbb{R}^{m} \times \mathbb{R}^{n-1}$ the map $\xi^{i} \rightarrow$ $F\left(x, \xi^{1}, \ldots, \xi^{i}, \ldots, \xi^{n}\right)$ belongs to $C^{2}(\mathbb{R})$, can be weakened requiring that the map $\xi^{i} \rightarrow$ $F\left(x, \xi^{1}, \ldots, \xi^{i}, \ldots, \xi^{n}\right)$ is only $C_{l o c}^{1,1}(\mathbb{R})$. Notice that the extension to locally Lipschitz nonlinearities could be very interesting from a physical viewpoint; indeed, very often, physical applications are run by locally Lipschitz forces.

A.2. On the F-monotonicity condition. Proceeding in our discussion about the consistency of assumptions made in Section 1, it is worth noticing that, as pointed out in [23], the notion of $F$-monotonicity (see Definition 1.2) seems to be crucial in order prove that a solution is one-dimensional. Indeed, let us consider the following system

$$
-\Delta u+\nabla F(u)=0 \quad \text { in } \mathbb{R}^{2},
$$


where $F: \mathbb{R}^{2} \longrightarrow \mathbb{R}$ is defined by:

$$
F\left(x_{1}, x_{2}\right):=\left(x_{1}-1\right)^{2} x_{2}^{2}+\left(x_{2}^{2}-1\right)^{2} .
$$

Then, $F$ does not satisfy condition $i i$ ) in the Definition 1.2 , indeed

$$
F_{12}\left(x_{1}, x_{2}\right)=4 x_{2}\left(x_{1}-1\right) .
$$

Moreover,

$$
\begin{aligned}
& F \in C^{2}\left(\mathbb{R}^{2}\right), \quad F((1,1))=0, \quad F((1,-1))=0, \quad F(\xi)>0 \quad \text { for } \xi \neq(1,1),(1,-1), \\
& \nabla^{2} F((1,1)) \geq I, \quad \nabla^{2} F((1,-1)) \geq I \\
& \nabla F(\xi) \cdot \xi \geq 0 \quad \text { for }|\xi| \geq R_{0}, \quad \text { for some } R_{0}>1,
\end{aligned}
$$

which, by [1, Theorem 1.1], imply that there exist entire solutions $\left(u^{1}, u^{2}\right)$ of (38) which are not one-dimensional.

A.3. Minimizers and stable solutions. We point out some conditions which ensure the validity of (10). As mentioned in the introduction, the system in (10) is associated to a suitable energy functional. Precisely, let us define

$$
\lambda_{1}^{i}(x, t):=\frac{\partial a_{i}}{\partial t}(x, t) t+a_{i}(x, t), \quad \lambda_{2}^{i}(x, t):=a_{i}(x, t), \quad i=1, \ldots, n,
$$

and

$$
\Lambda_{2}^{i}(x, t):=\int_{0}^{t} \lambda_{2}^{i}(x,|\tau|) \tau \mathrm{d} \tau .
$$

Then, it a is a matter of computations that the energy functional related to (1) is

$$
I_{\Omega}\left(u^{1}, \ldots, u^{n}\right):=\sum_{i=1}^{n} \int_{\Omega} \Lambda_{2}^{i}\left(x,\left|\nabla u^{i}\right|\right)-F\left(x, u^{1}, \ldots, u^{n}\right) .
$$

According to [12], we give the following definition:

Definition A.2. A family $\left(u^{1}, \ldots, u^{n}\right)$ is said to be a local minimizer for $I_{\Omega}$ if, for any bounded open set $U \subset \Omega, I_{U}\left(u^{1}, \ldots, u^{n}\right)$ is well-defined and finite, and

$$
I_{U}\left(u^{1}+\psi^{1}, \ldots, u^{n}+\psi^{n}\right) \geq I_{U}\left(u^{1}, \ldots, u^{n}\right)
$$

for any $\left(\psi^{1}, \ldots, \psi^{n}\right) \in C_{c}^{\infty}\left(U, \mathbb{R}^{n}\right)$.

The following lemma is the exact counterpart for systems of the result proved in [12, Lemma B.1] for the case of one equation.

Lemma A.3. Let $\Omega \subset \mathbb{R}^{N}$ be an open set. If $\left(u^{1}, \ldots, u^{n}\right)$ is a local minimizer of $I_{\Omega}$, then $\left(u^{1}, \ldots, u^{n}\right)$ is a weak solution of (1) and satisfies (7).

Proof. We start proving that every local minimizer $u=\left(u^{1}, \ldots, u^{n}\right)$ of $I_{\Omega}$ is a weak solution of (1). To this end, let $U \subset \Omega$ be open and bounded and consider $\psi \in C_{c}^{\infty}(U)$. Then, for every $i=1, \ldots, n$, we get

$$
\left.\frac{\mathrm{d}}{\mathrm{d} s}\right|_{s=0} I_{U}\left(u^{1}, \ldots, u^{i}+s \psi, \ldots, u^{n}\right)=0
$$


and, recalling the definition of $I_{U}$ in (39),

$$
\int_{\Omega} a_{i}\left(x,\left|\nabla u^{i}\right|(X)\right)\left\langle\nabla u^{i}(X), \nabla \psi(X)\right\rangle \mathrm{d} X=\int_{\Omega} F_{i}\left(x, u^{1}, \ldots, u^{n}\right) \psi(X) \mathrm{d} X,
$$

which is the first part of the thesis.

Finally, for every $\psi=\left(\psi^{1}, \ldots, \psi^{n}\right) \in C_{c}^{\infty}\left(U, \mathbb{R}^{n}\right)$,

$$
\begin{aligned}
0 \leq & \left.\frac{\mathrm{d}^{2}}{\mathrm{~d} s^{2}}\right|_{s=0} I_{U}\left(u^{1}+s \psi^{1}, \ldots, u^{n}+s \psi^{n}\right) \\
= & \left.\sum_{i=1}^{n} \frac{\mathrm{d}}{\mathrm{d} s}\right|_{s=0} \int_{\Omega}\left(a_{i}\left(x,\left|\nabla u^{i}+s \nabla \psi^{i}\right|(X)\right)\left\langle\nabla u^{i}+s \nabla \psi^{i}, \nabla \psi^{i}\right\rangle\right. \\
& \left.\quad-F_{i}\left(x, u^{1}+s \psi^{1}, \ldots, u^{n}+s \psi^{n}\right) \psi^{i}(X)\right) \mathrm{d} X \\
= & \sum_{i=1}^{n} \int_{\Omega}\left\langle\mathcal{A}^{i}\left(x, \nabla u^{i}(X)\right) \nabla \psi^{i}(X), \nabla \psi^{i}(X)\right\rangle \mathrm{d} X \\
& \quad-\sum_{i, j=1}^{n} \int_{\Omega} F_{i j}\left(x, u^{1}, \ldots, u^{n}\right) \psi^{i}(X) \psi^{j}(X) \mathrm{d} X,
\end{aligned}
$$

and the proof is accomplished.

In the following proposition we give a sufficient condition for the assumption (10) to hold for local minimizers.

Proposition A.4. Let $N \leq 3$, and assume that, for each $i=1, \ldots, n$, there exists $C_{i}>0$ such that

$$
\begin{aligned}
& \lambda_{1}^{i}(x, t)>0, \quad \forall x \in \mathbb{R}^{m}, t \in(0,+\infty), \\
& \lambda_{1}^{i}(x, t) t^{2}, \lambda_{2}^{i}(x, t) t^{2} \leq C_{i} \Lambda_{2}^{i}(x, t), \quad \forall x \in \mathbb{R}^{m}, t \in\left(0, C_{i}\right] .
\end{aligned}
$$

Moreover, we assume that for all $x \in \mathbb{R}^{m}$, and $s, t \in[0,+\infty)$

$$
\begin{aligned}
& \Lambda_{2}^{i}(x, s) \geq 0, \\
& \Lambda_{2}^{i}(x, s+t) \leq \bar{C}_{i}\left[\Lambda_{2}^{i}(x, s)+\Lambda_{2}^{i}(x, t)\right], \\
& \Lambda_{2}^{i}(x, s) \leq \alpha_{i}(x) g_{i}(s),
\end{aligned}
$$

for some $\bar{C}_{i}>0, \alpha_{i} \in L_{\text {loc }}^{\infty}\left(\mathbb{R}^{m}\right)$ and $g_{i}:[0,+\infty) \longrightarrow \mathbb{R}$ monotone increasing. Finally, for all $x \in \mathbb{R}^{m}$ and $\xi \in \mathbb{R}^{n}$, we suppose that the following holds

$$
F(x, \xi)=0, \quad \forall x \in \mathbb{R}^{m}, \forall \xi \in \mathbb{S}^{n-1},
$$

and

$$
\sup _{x \in \mathbb{R}^{m},|\xi| \leq 1}|F(x, \xi)| \leq+\infty .
$$

Then, assumption (10) is satisfied for every local minimizer $\left(u^{1}, \ldots, u^{n}\right)$ of $I_{\mathbb{R}^{N}}$ such that $\left|u^{i}\right|+\left|\nabla u^{i}\right| \leq M, i=1, \ldots, n$. 
Proof. We start observing that, for each $i=1, \ldots, n$, and $x \in \mathbb{R}^{m}$, if $\xi, v \in \mathbb{R}^{N}$ with $|v| \leq 1$ and $|\xi| \leq M$, then

$$
|\xi|^{2}\left\langle\mathcal{A}^{i}(x, \xi) v, v\right\rangle \leq C_{M} \Lambda_{2}^{i}(x,|\xi|) .
$$

Indeed, by a simple calculation we get

$$
\begin{aligned}
|\xi|^{2}\left\langle\mathcal{A}^{i}(x, \xi) v, v\right\rangle & =\frac{\partial a_{i}}{\partial t}(x,|\xi|)|\xi|\langle\xi, v\rangle^{2}+a_{i}(x,|\xi|)|v|^{2}|\xi|^{2} \\
& =\lambda_{1}^{i}(x,|\xi|)\langle\xi, v\rangle^{2}+\lambda_{2}^{i}(x,|\xi|)\left[|v|^{2}|\xi|^{2}-\langle\xi, v\rangle^{2}\right] \\
& \leq\left(\lambda_{1}^{i}(x,|\xi|)+\lambda_{2}^{i}(x,|\xi|)\right)|v|^{2}|\xi|^{2} \\
& \leq C_{M} \Lambda_{2}^{i}(x,|\xi|),
\end{aligned}
$$

where in the last inequality we have used (41) and the fact that $|v| \leq 1$.

Let $R>1$ and take $\psi=\left(\psi^{1}, \ldots, \psi^{n}\right) \in\left(C_{c}^{\infty}\left(\mathbb{R}^{N}\right)\right)^{n}$ with the property that, for each $i=1, \ldots, n, \psi^{i}=-1$ in $B_{R-1}, \psi^{i}=1$ on $\partial B_{R}$ and $\left|\nabla \psi^{i}\right| \leq M$ in $B_{R} \backslash B_{R-1}$. Let us define

$$
v^{i}(X):=\min \left\{u^{i}(X), \psi^{i}(X)\right\}, \quad i=1, \ldots, n,
$$

and observe that, by the minimality of $u$ and (45),

$$
\sum_{i=1}^{n} \int_{B_{R}} \Lambda_{2}^{i}\left(x,\left|\nabla u^{i}\right|\right) \leq I_{B_{R}}\left(u^{1}, \ldots, u^{n}\right) \leq I_{B_{R}}\left(v^{1}, \ldots, v^{n}\right) .
$$

By (42), (46), (43) and (47) we get

$$
\begin{aligned}
\sum_{i=1}^{n} \int_{B_{R}} \Lambda_{2}^{i}\left(x,\left|\nabla u^{i}\right|\right) & \leq \int_{B_{R} \backslash B_{R-1}} \sum_{i=1}^{n} \Lambda_{2}^{i}\left(x,\left|\nabla v^{i}\right|\right)-F\left(x, v^{1}, \ldots, v^{n}\right) \\
& \leq \max \left\{\bar{C}_{i}\right\} \int_{B_{R} \backslash B_{R-1}} \sum_{i=1}^{n}\left(\Lambda_{2}^{i}\left(x,\left|\nabla u^{i}\right|\right)+\Lambda_{2}^{i}\left(x,\left|\nabla \psi^{i}\right|\right)\right)+\sup _{\mathbb{R}^{m} \times Q}|F|,
\end{aligned}
$$

where $Q:=[-1,1] \times \cdots \times[-1,1]$ is the cube in $\mathbb{R}^{n}$. Using (44), we obtain

$$
\begin{aligned}
\sum_{i=1}^{n} \int_{B_{R}} \Lambda_{2}^{i}\left(x,\left|\nabla u^{i}\right|\right) & \leq \max \left\{\bar{C}_{i}\right\} \int_{B_{R} \backslash B_{R-1}} \sum_{i=1}^{n} \alpha_{i}(x)\left(g_{i}\left(\left|\nabla u^{i}\right|\right)+g_{i}\left(\left|\nabla \psi^{i}\right|\right)\right)+\sup _{\mathbb{R}^{m} \times Q}|F| \\
& \leq 2 \max \left\{\bar{C}_{i}\right\} \int_{B_{R} \backslash B_{R-1}} \sum_{i=1}^{n} g_{i}(M) \sup _{x} \alpha_{i}+\sup _{\mathbb{R}^{m} \times Q}|F| \\
& \leq C R^{N-1},
\end{aligned}
$$

for some $C>0$. Finally, thanks to (48), (49) and the fact that $\mathcal{A}^{i}$ is positive definite, we have

$$
\int_{B_{R}}\left|\nabla u^{i}\right|^{2}\left\langle\mathcal{A}^{i}\left(x, \nabla u^{i}\right) v, v\right\rangle \leq C R^{N-1}
$$

and, taking as $v$ the normalized eigenvector corresponding to $\overline{\mathcal{A}}^{i}$, we get the thesis. 
We conclude this Appendix providing an example of functional which satisfies the hypotheses in Proposition A.4, and hence the assumption (10), obtaining, from Theorem 1.5, the one-dimensional symmetry for local minimizers.

Corollary A.5. Let $N \leq 3$, and let $\alpha \in L^{\infty}\left(\mathbb{R}^{m}\right)$ be strictly positive, and $F \in C^{2}\left(\mathbb{R}^{2}\right)$ such that $G:=\alpha F$ satisfies (45)-(47). Suppose that $F_{12}$ does not change sign.

For every $p_{1}, p_{2} \in(1,3)$, let us define the functional

$$
I_{\mathbb{R}^{N}}:=\int_{\mathbb{R}^{N}}\left|\nabla u^{1}(X)\right|^{p_{1}}+\left|\nabla u^{2}(X)\right|^{p_{2}}-\alpha(x) F\left(u^{1}(X), u^{2}(X)\right) \mathrm{d} X .
$$

Then, for every local minimizer $\left(u^{1}, u^{2}\right)$ of $I_{\mathbb{R}^{N}}$ such that $u^{1} \in W^{1, p_{1}}\left(\mathbb{R}^{N}\right) \cap L^{\infty}\left(\mathbb{R}^{N}\right)$, $u^{2} \in W^{1, p_{2}}\left(\mathbb{R}^{N}\right) \cap L^{\infty}\left(\mathbb{R}^{N}\right)$, with $\left|\nabla u^{1}\right|,\left|\nabla u^{2}\right| \in L^{\infty}\left(\mathbb{R}^{N}\right)$, there exist $u_{0}^{1}, u_{0}^{2}: \mathbb{R}^{m} \times \mathbb{R} \longrightarrow \mathbb{R}$ and $\omega_{1}, \omega_{2}: \mathbb{R}^{m} \rightarrow \mathbb{S}^{N-m-1}$ such that

$$
u^{1}(x, y)=u_{0}^{1}\left(x,\left\langle\omega_{1}(x), y\right\rangle\right), \quad u^{2}(x, y)=u_{0}^{2}\left(x,\left\langle\omega_{2}(x), y\right\rangle\right),
$$

for any $(x, y) \in \mathbb{R}^{m} \times \mathbb{R}^{N-m}$. Moreover, $\omega_{i}, i=1,2$, is constant in any connected component of $\left\{\nabla_{y} u^{i} \neq 0\right\}$.

Proof. The proof easily follows from Theorem 1.5 and Proposition A.4. Indeed,

$$
\Lambda_{2}^{1}(x, t)=|t|^{p_{1}}, \quad \Lambda_{2}^{2}(x, t)=|t|^{p_{2}}
$$

satisfy conditions (42), (43), (44) and

$$
\begin{aligned}
& \lambda_{1}^{1}(x, t)=\left(p_{1}-1\right)|t|^{p_{1}-2}, \quad \lambda_{1}^{2}(x, t)=\left(p_{2}-1\right)|t|^{p_{2}-2}, \\
& \lambda_{2}^{1}(x, t)=|t|^{p_{1}-2}, \quad \lambda_{2}^{2}(x, t)=|t|^{p_{2}-2}
\end{aligned}
$$

satisfy (40) and (41). Moreover, as proved in [11], both $\mathcal{A}^{1}, \mathcal{A}^{2}$ are positive definite for every $p_{1}, p_{2}>1$ and satisfy (6) when $p_{1}, p_{2} \geq 2$, and even for $p_{1}, p_{2}>1$ as long as $\left\{\nabla u^{1}=0\right\}=\left\{\nabla u^{2}=0\right\}=\varnothing$.

\section{ACKNOWLEDGMENTS}

The authors would like to thank Enrico Valdinoci for his very deep and useful comments and suggestions.

\section{REFERENCES}

[1] Alama, S., Bronsard, L., Gui, C.: Stationary layered solutions in $\mathbb{R}^{2}$ for an Allen-Cahn system with multiple-well potential, Calc. Var. Partial Differential Equations 5, 359-390 (1997).

[2] Alberti, G., Ambrosio, L., Cabré, X.: On a long-standing conjecture of E. De Giorgi: symmetry in 3D for general nonlinearities and a local minimality property, Acta Appl. Math. 65 (1-3), 9-33 (2001).

[3] Ambrosio, L., Cabré, X.: Entire solutions of semilinear elliptic equations in $\mathbb{R}^{3}$ and a conjecture of De Giorgi, J. Amer. Math. Soc. 13, 725-739 (2000).

[4] Berestycki, H., Caffarelli, L., Nirenberg, L.: Further qualitative properties for elliptic equations in unbounded domains, Ann. Sc. Norm. Super. Pisa Cl. Sci.(4) 25, no. 1-2 (1998), 69-94, 1997 dedicated to Ennio De Giorgi.

[5] Berestycki, H., Lin. T.C., Wei, J., Zhao, C.: On phase-separation model: Asymptotics and qualitative properties, to appear in Arch. Ration. Mech. Anal., available at http://lodel.ehess.fr/cams/docannexe.php?id=1192 
[6] Berestycki, H., Terracini, S., Wang, K., Wei, J.: On entire solutions of an elliptic system modeling phase separations, preprint (2012), available at http://arxiv.org/abs/1204.1038

[7] Cabré, X., Cinti, E.: Sharp energy estimates for nonlinear fractional diffusion equations, preprint (2012), available at http://arxiv.org/abs/1207.6194

[8] Cabré, X., Solà-Morales, J.: Layer solutions in a half-space for boundary reactions, Comm. Pure Appl. Math. 58, no. 12, 1678-1732 (2005).

[9] Cabré, X., Sire, Y.: Nonlinear equations for fractional Laplacians I: regularity, maximum principles, and Hamiltonian estimates, preprint (2010), available at http://arxiv.org/abs/1012.0867

[10] Cabré, X., Sire, Y.: Nonlinear equations for fractional Laplacians II: existence, uniqueness, and qualitative properties of solutions, preprint (2011), available at http://arxiv.org/abs/1111.0796

[11] Chermisi, M., Valdinoci, E.: A symmetry result for a general class of divergence form PDEs in fibered media. Nonlinear Anal. 73, 695-703 (2010).

[12] Chermisi, M., Valdinoci, E.: Fibered nonlinearities for $p(x)$-Laplace equations, Adv. Calc. Var. 2, 185-205 (2009).

[13] Damascelli, L., Sciunzi, B.: Regularity, monotonicity and symmetry of positive solutions of m-Laplace equations, J. Differential Equations 206, no. 1, 483-515 (2004).

[14] Del Pino, M., Kowalczyk, M., Wei, J.: On De Giorgi's conjecture in dimension $N \geq 9$, Ann. of Math. (2) 174, no. 3, 1485-1569 (2011).

[15] De Giorgi, E.: Convergence problems for functionals and operators, in Proceedings of the International Meeting on Recent Methods in Nonlinear Analysis (Rome, 1978), pp. 131-188, Pitagora, Bologna (1979).

[16] DiBenedetto, E.: $C^{1, \alpha}$ local regularity of weak solutions of degenerate elliptic equations, Nonlinear Anal. 7, no. 8, 827-850 (1983).

[17] Dipierro, S.: Geometric inequalities and symmetry results for elliptic systems, to appear in Discrete Contin. Dyn. Syst., available at http://arxiv.org/pdf/1207.4435.pdf

[18] Dipierro, S., Pinamonti, A.: A geometric inequality and a symmetry result for elliptic systems involving the fractional Laplacian, preprint (2012), available at http://arxiv.org/abs/1211.2622

[19] Farina, A.: Propriétés Qualitatives de Solutions d'équations et Systémes d'équations Non-linéaires, Habilitation a Diriger des Recherches, Paris VI (2002).

[20] Farina, A., Sciunzi, B., Valdinoci, E.: Bernstein and De Giorgi type problems: new results via a geometric approach, Ann. Sc. Norm. Super. Pisa Cl. Sci. (5) 7, 741-791 (2008).

[21] Farina, A., Sire, Y., Valdinoci, E.: Stable solutions of elliptic equations on Riemannian manifolds, to appear in J. Geom. Anal., available at http://arxiv.org/abs/0809.3025

[22] Farina, A., Sire, Y., Valdinoci, E.: Stable solutions of elliptic equations on Riemannian manifolds with Euclidean coverings, Proc. Amer. Math. Soc. 140, no. 3, 927-930 (2012).

[23] Fazly, M., Ghoussoub, N.: De Giorgi type results for elliptic systems, to appear in Calc. Var. Partial Differential Equations, available at http://arxiv.org/abs/1203.6114

[24] Ghoussoub, N., Gui, C.: On a conjecture of De Giorgi and some related problems, Math. Ann. 311, no. 3, 481-491 (1998).

[25] Ghoussoub, N., Gui, C.: On De Giorgi's conjecture in dimensions 4 and 5, Ann. of Math. (2) 157, no. 1, 313-334 (2003).

[26] Ladyzenskaja, O.A., Ural'tzeva N.N.: Linear and Quasilinear Elliptic Equations, Academic Press, New York, (1968).

[27] Lewis, J.L.: Regularity of the derivatives of solutions to certain degenerate elliptic equations, Indiana Univ. Math. J. 32, no. 6, 849-858 (1983).

[28] Lieb, E.H., Loss, M.: Analysis, in: Graduate Studies in Mathematics, vol. 14, American Mathematical Society, Providence, RI (1997).

[29] Noris, B., Tavares, H., Terracini, S., Verzini, G.: Uniform Hölder bounds for nonlinear Schrödinger systems with strong competition, Comm. Pure Appl. Math. 63, 267-302 (2012).

[30] Pinamonti, A., Valdinoci, E.: A geometric inequality for stable solutions of semilinear elliptic problems in the Engel group, Ann. Acad. Sci. Fenn. Math. 37, 357-373 (2012).

[31] Savin, O.: Regularity of flat level sets in phase transitions, Ann. of Math. (2) 169 (1), 41-78 (2009). 
[32] Savin, O., Valdinoci, E.: Elliptic PDEs with fibered nonlinearities, J. Geom. Anal. 19, no. 2, 420-432 (2008).

[33] Sire, Y., Valdinoci, E.: Fractional Laplacian phase transitions and boundary reactions: A geometric inequality and a symmetry result, J. Funct. Anal. 256, 1842-1864 (2009).

[34] Sternberg, P., Zumbrun, K.: A Poincaré inequality with applications to volume-constrained areaminimizing surfaces, J. Reine Angew. Math. 503, 63-85 (1998).

[35] Sternberg, P., Zumbrun, K.: Connectivity of phase boundaries in strictly convex domains, Arch. Ration. Mech. Anal. 141, 375-400 (1998).

[36] Tolksdorf, P.: Regularity for a more general class of quasilinear elliptic equations, J. Differential Equations 51, no. 1, 126-150 (1984).

[37] Wang, K.: On the De Giorgi type conjecture for an elliptic system modeling phase separation, preprint (2012), available at http://arxiv.org/abs/1207.5285

Departamento de Ingeniería Matemática, Universidad de Chile, Casilla 170 Correo 3 , Santiago, Chile

E-mail address: sdipierro@dim.uchile.cl

Dipartimento di Matematica, Università di Padova, Via Trieste 63, Padova, Italia

E-mail address: pinamonti@science.unitn.it 